\title{
Value of CDX2, villin, and $\alpha$-methylacyl coenzyme $A$ racemase immunostains in the distinction between primary adenocarcinoma of the bladder and secondary colorectal adenocarcinoma
}

\author{
Namsoo Suh ${ }^{1}$, Ximing J Yang ${ }^{2}$, Maria S Tretiakova ${ }^{3}$, Peter A Humphrey ${ }^{1}$ and \\ Hanlin L Wang ${ }^{1}$ \\ ${ }^{1}$ Lauren V Ackerman Laboratory of Surgical Pathology, Department of Pathology and Immunology, \\ Washington University School of Medicine, St Louis, MO, USA; ${ }^{2}$ Department of Pathology, Northwestern \\ University Feinberg School of Medicine, Chicago, IL, USA and ${ }^{3}$ Department of Pathology, University of \\ Chicago Hospitals, Chicago, IL, USA
}

\begin{abstract}
Primary adenocarcinoma of the urinary bladder is an uncommon neoplasm that can be indistinguishable morphologically from colorectal adenocarcinoma secondarily involving the bladder by direct extension or metastasis. In the current study, 17 enteric-type primary adenocarcinomas of the bladder were immunohistochemically examined for the expression of CDX2, villin and $\alpha$-methylacyl coenzyme A racemase (AMACR), immunomarkers preferentially expressed in colorectal adenocarcinoma. For comparison, 17 secondary colorectal adenocarcinomas involving the bladder, 23 primary colorectal adenocarcinomas and 14 conventional urothelial carcinomas were similarly studied. The results show that all $40(100 \%)$ colorectal adenocarcinomas expressed CDX2 and 39 (98\%) expressed villin. The expression of these two immunomarkers was less frequent in primary bladder adenocarcinomas, observed in eight $(47 \%)$ and $11(65 \%)$ cases, respectively $(P<0.0001$ and $\boldsymbol{P}=0.0019$, respectively). The frequency of positive AMACR immunostaining was similar between these two types of tumors, detected in 28 (70\%) colorectal adenocarcinomas and 11 (65\%) primary bladder adenocarcinomas $(P=0.694)$. None of the urothelial carcinomas exhibited CDX2 or villin immunoreactivity; and only two (14\%) showed positive staining for AMACR. These results demonstrate that CDX2 and villin are of diagnostic value in aiding in the distinction between primary adenocarcinoma of the bladder and secondary colorectal carcinoma. Lack of CDX2 and villin signals points strongly to a bladder primary.
\end{abstract}

Modern Pathology (2005) 18, 1217-1222. doi:10.1038/modpathol.3800407; published online 1 April 2005

Keywords: urinary bladder; colon; rectum; adenocarcinoma; CDX2; villin; $\alpha$-methylacyl coenzyme A racemase

Secondary involvement of the urinary bladder by adenocarcinoma from other anatomic sites, either by direct extension or hematogenous/lymphatic metastasis, occurs more frequently than adenocarcinoma originating from the bladder itself. ${ }^{1}$ The distinction between primary and secondary adenocarcinomas of the bladder is important to clinical management, but

Correspondence: Dr HL Wang, MD, PhD, Lauren V Ackerman Laboratory of Surgical Pathology, Department of Pathology and Immunology, Campus Box 8118, Washington University School of Medicine, 660 South Euclid Avenue, St Louis, MO 63110-1093, USA.

E-mail: hwang@path.wustl.edu

Received 5 January 2005; accepted 31 January 2005; published online 1 April 2005 may not always be straightforward to pathologists or clinicians. This is particularly true when the secondary adenocarcinoma is of colorectal origin, which may exhibit histologic features similar to or indistinguishable from primary bladder adenocarcinoma.

We have previously reported that a panel of immunomarkers, including $\beta$-catenin, cytokeratin (CK) 7, CK20 and thrombomodulin, is of diagnostic value in helping distinguishing primary bladder adenocarcinoma from secondary adenocarcinoma of the colorectal origin. ${ }^{2}$ Primary bladder adenocarcinoma was shown to express a distinct immunophenotype that was intermediate between urothelial carcinoma and colorectal adenocarcinoma. In comparison with colorectal adenocarcinoma, primary 
bladder adenocarcinoma expressed CK7 and thrombomodulin more frequently, CK20 less frequently, and did not exhibit nuclear staining for $\beta$-catenin. In this study, we examined additional immunomarkers, CDX2, villin and $\alpha$-methylacyl coenzyme A racemase (AMACR) that have been shown to be preferentially expressed in colorectal adenocarcinoma, to assess their potential diagnostic value in the distinction between primary adenocarcinoma of the bladder and secondary involvement by colorectal adenocarcinoma.

\section{Materials and methods}

\section{Case Selection}

A total of 17 enteric-type primary adenocarcinomas of the urinary bladder were retrieved from the 19892003 surgical pathology archives at Washington University Barnes-Jewish Hospital and the 19862000 surgical pathology archives at the University of Chicago Hospitals. Clinical data were reviewed to ensure that they were indeed bladder primaries. Cases with any known history of adenocarcinoma in other organs were excluded from this study. All the tumors were derived from the bladder proper and morphologically reminiscent of well or moderately differentiated colorectal adenocarcinomas. None of the cases was associated with Schistosoma haematobium infestation or exstrophy. Hematoxylin and eosin (H\&E)-stained slides were reviewed to confirm the diagnosis and to exclude conventional urothelial carcinomas with focal glandular differentiation.

A total of 40 cases of colorectal adenocarcinoma were selected for the study. This included 17 cases secondarily involving the bladder by direct extension based on clinical history. The remaining 23 cases were colorectal primaries randomly selected from the left and right colon. In addition, 14 conventional urothelial carcinomas (without glandular differentiation) were included for comparison.

\section{Immunohistochemistry}

Immunohistochemical studies were performed on 4$\mu \mathrm{m}$ tissue sections employing the LSAB Plus system obtained from DAKO Corp. (Carpinteria, CA, USA) and the ABC kit obtained from Vector Laboratories (Burlingame, CA, USA), following the manufacturers' instructions with slight modifications. Briefly, deparaffinized tissue sections were first treated with $3 \% \mathrm{H}_{2} \mathrm{O}_{2}$ for $15 \mathrm{~min}$ to inhibit endogenous peroxidase followed by antigen retrieval as described below. After incubation with blocking serum for $20 \mathrm{~min}$, sections were incubated with the primary antibodies (described below) for $1 \mathrm{~h}$ at room temperature, followed by further incubation with biotinylated link antibody and peroxidase-labeled streptavidin. The staining was developed by reaction with diaminobenzidine substrate-chromogen solution followed by counterstaining with hematoxylin 7211 purchased from Richard-Allan Scientific (Kalamazoo, MI, USA).

The primary antibodies used in this study included mouse anti-CDX2 IgG1 (clone CDX2-88) obtained from BioGenex (San Ramon, CA, USA) used at 1:50 dilution, mouse anti-villin IgG1 (clone ID2C3) obtained from Immunotech (Marseille, France) used at 1:80 dilution, and prediluted rabbit anti-AMACR IgG obtained from Biocare Medical (Walnut Creek, CA, USA). The antigen retrieval conditions were microwave heating in $10 \mathrm{mM}$ citrate buffer ( $\mathrm{pH} \mathrm{6.0)} \mathrm{for} 10 \mathrm{~min}$ for CDX2, 8 min for villin and $20 \mathrm{~min}$ for AMACR. In each experiment, a negative control was included in which the primary antibody was replaced by preimmune mouse or rabbit IgG. Positive controls used in this study were sections from a prostate that contained both adenocarcinoma and benign prostatic glands for AMACR, and a colorectal adenocarcinoma known to be positive for CDX2 and villin.

A tumor was recorded positive if greater than $5 \%$ of the tumor cells exhibited nuclear staining for CDX2, apical and/or cytoplasmic staining for villin, and cytoplasmic staining for AMACR. The positivity was stratified as diffuse (greater than $75 \%$ of the tumor cells stained) and focal (5-75\%) which was further divided into three subgroups $(5-25,26-50$ and $51-75 \%$ ).

\section{Statistical Analysis}

Statistical analysis was performed using the Statistica software for windows (Tulsa, OK, USA). A $P$ value of $<0.05$, as determined by two-tailed Fisher's exact test or the $\chi^{2}$-test with Yates continuity correction, was considered statistically significant.

\section{Results}

Table 1 compares the immunohistochemical findings in bladder and colorectal carcinomas. Specifically, all 40 colorectal adenocarcinomas (primary and secondary) exhibited positive nuclear staining for CDX2 (100\%), with a strong and diffuse staining pattern (Figure 1a) seen in 37 cases (93\%). This was in contrast to primary bladder adenocarcinomas where only eight of 17 cases $(47 \%)$ showed nuclear CDX2 immunoreactivity $(P<0.0001)$. Among them, four cases showed a focal staining pattern (Figure 1b); diffuse positivity was observed in only four cases $(24 \%)$. None of the 14 urothelial carcinomas exhibited CDX2 immunoreactivity.

Similar to CDX2, villin was also universally expressed in colorectal adenocarcinomas (Figure 2a), with only one exception of a secondary tumor where positive staining was not detected. In total, 11 cases $(65 \%)$ of primary bladder adenocarcinoma exhibited a variable degree of villin immunoreactivity (Figure 2b). Although the difference did not 
Table 1 Comparison of CDX2, villin and AMACR expression among primary bladder adenocarcinoma (PBA), secondary colorectal adenocarcinoma (SCA), primary colorectal adenocarcinoma (PCA), and urothelial carcinoma (UC)

\begin{tabular}{|c|c|c|c|c|c|}
\hline \multicolumn{2}{|c|}{ Marker Immunoreactivity } & \multicolumn{4}{|c|}{ No. (\%) positive } \\
\hline & & $\begin{array}{c}P B A \\
(\mathrm{n}=17)\end{array}$ & $\begin{array}{c}S C A \\
(\mathrm{n}=17)\end{array}$ & $\begin{array}{c}P C A \\
(\mathrm{n}=23)\end{array}$ & $\begin{array}{c}U C \\
(\mathrm{n}=14)\end{array}$ \\
\hline \multirow[t]{5}{*}{ CDX2 } & Negative $(<5 \%)$ & $9(53)$ & 0 & 0 & $14(100)$ \\
\hline & $5-25 \%$ & 0 & 0 & 0 & 0 \\
\hline & $26-50 \%$ & 3 (18) & 0 & $1(4)$ & 0 \\
\hline & $51-75 \%$ & $1(6)$ & $1(6)$ & $1(4)$ & 0 \\
\hline & $>75 \%$ & $4(24)$ & $16(94)$ & $21(91)$ & 0 \\
\hline \multirow{5}{*}{ Villin } & Negative $(<$ & $6(35)$ & 1( & 0 & $14(100)$ \\
\hline & $5-25 \%$ & $1(6)$ & $2(12)$ & 0 & 0 \\
\hline & $26-50 \%$ & 2 (12) & $1(6)$ & 0 & 0 \\
\hline & $51-75 \%$ & $5(29)$ & $4(24)$ & $1(4)$ & 0 \\
\hline & $>75 \%$ & $3(18)$ & $9(53)$ & $22(96)$ & 0 \\
\hline & $6(3$ & 4( & $8(35)$ & $12(86)$ \\
\hline \multicolumn{2}{|c|}{$\begin{array}{l}\text { AMACRNegative }(<5 \%) \\
5-25 \%\end{array}$} & $2(12)$ & 3 (18) & $1(4)$ & $1(7)$ \\
\hline & $26-50 \%$ & 3 (18) & $1(6)$ & $1(4)$ & 0 \\
\hline & $51-75 \%$ & $4(24)$ & $4(24)$ & 3 (13) & 0 \\
\hline & $>75 \%$ & 2 (12) & $5(29)$ & $10(44)$ & $1(7)$ \\
\hline
\end{tabular}

The $\%$ denotes the percentage of tumor cells positively stained.

reach the statistical significance when compared only with secondary colorectal adenocarcinoma $(P=0.0854)$, it was significant when primary colorectal adenocarcinomas were combined $(P=0.0019)$. Again, urothelial carcinomas were negative for villin expression.

The frequency of AMACR positivity was similar between bladder and colorectal adenocarcinomas, observed in $11(65 \%)$ and $28(70 \%)$ cases, respectively $(P=0.694)$. The staining patterns were also similar between these two types of tumors, with diffuse positivity (Figure 3a) seen in 12 and $38 \%$ of the cases, respectively $(P=0.052)$, and focal (Figure $3 \mathrm{~b})$ in the remaining cases. Only two urothelial carcinomas $(14 \%)$ showed positive staining for AMACR.

Table 2 shows that primary bladder adenocarcinomas less frequently coexpressed CDX2/villin, CDX2/AMACR and CDX2/villin/AMACR when compared with colorectal counterparts $(P<0.0001$, $P=0.008$ and $P=0.0023$, respectively). Bladder adenocarcinomas also appeared to less frequently coexpress villin/AMACR, but the difference from colorectal adenocarcinomas was not statistically significant $(P=0.1005)$.

In total, 12 primary bladder adenocarcinomas and nine secondary colorectal adenocarcinomas included in this study have been previously examined for the expression of CK7, CK20, thrombomodulin and $\beta$-catenin. ${ }^{2} \mathrm{~A}$ distinct expression pattern, characterized by CK7 and thrombomodulin negativity, CK20, CDX2 and villin positivity, and nuclear $\beta$ -

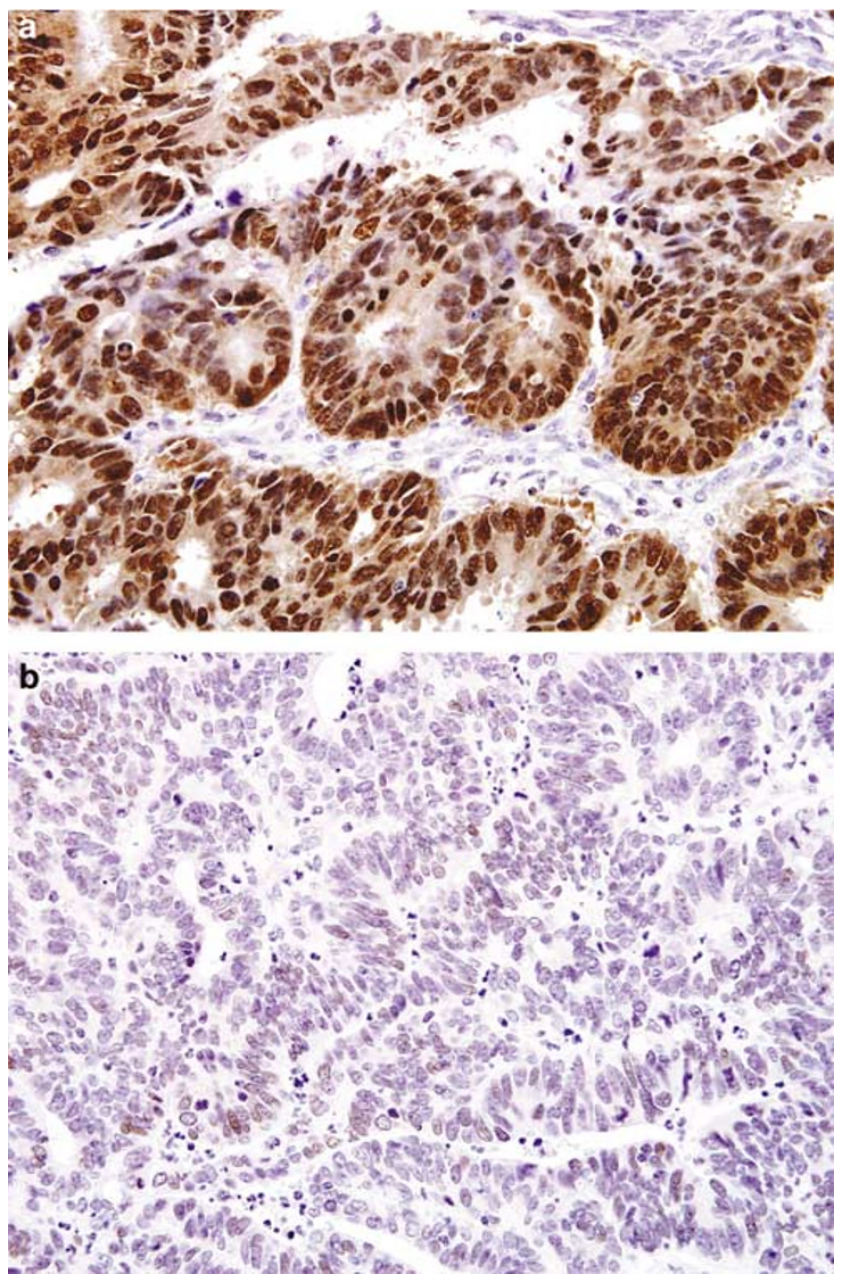

Figure 1 Strong and diffuse nuclear staining (with some cytoplasmic positivity) for CDX2 seen in a secondary colorectal adenocarcinoma (a). Note that essentially every tumor cell was positively stained in this case. The staining was focal and weaker in some of the primary bladder adenocarcinomas (b) (original magnification $\times 400$ ).

catenin localization, was observed in six secondary colorectal adenocarcinomas $(67 \%)$. This was in marked contrast to primary bladder adenocarcinomas where none of the cases studied showed this expression profile $(P=0.02)$.

\section{Discussion}

Primary adenocarcinoma occurs only rarely in the urinary bladder, accounting for $0.5-2 \%$ of all primary bladder tumors. ${ }^{3}$ Therefore, the possibility of secondary involvement should always be considered when an adenocarcinoma is encountered in the bladder. In this regard, colorectal adenocarcinoma is of great importance not only because it is the most common secondary tumor involving the bladder, ${ }^{1}$ but also because of its morphological similarity to primary bladder adenocarcinoma. The distinction between these two types of tumors can 

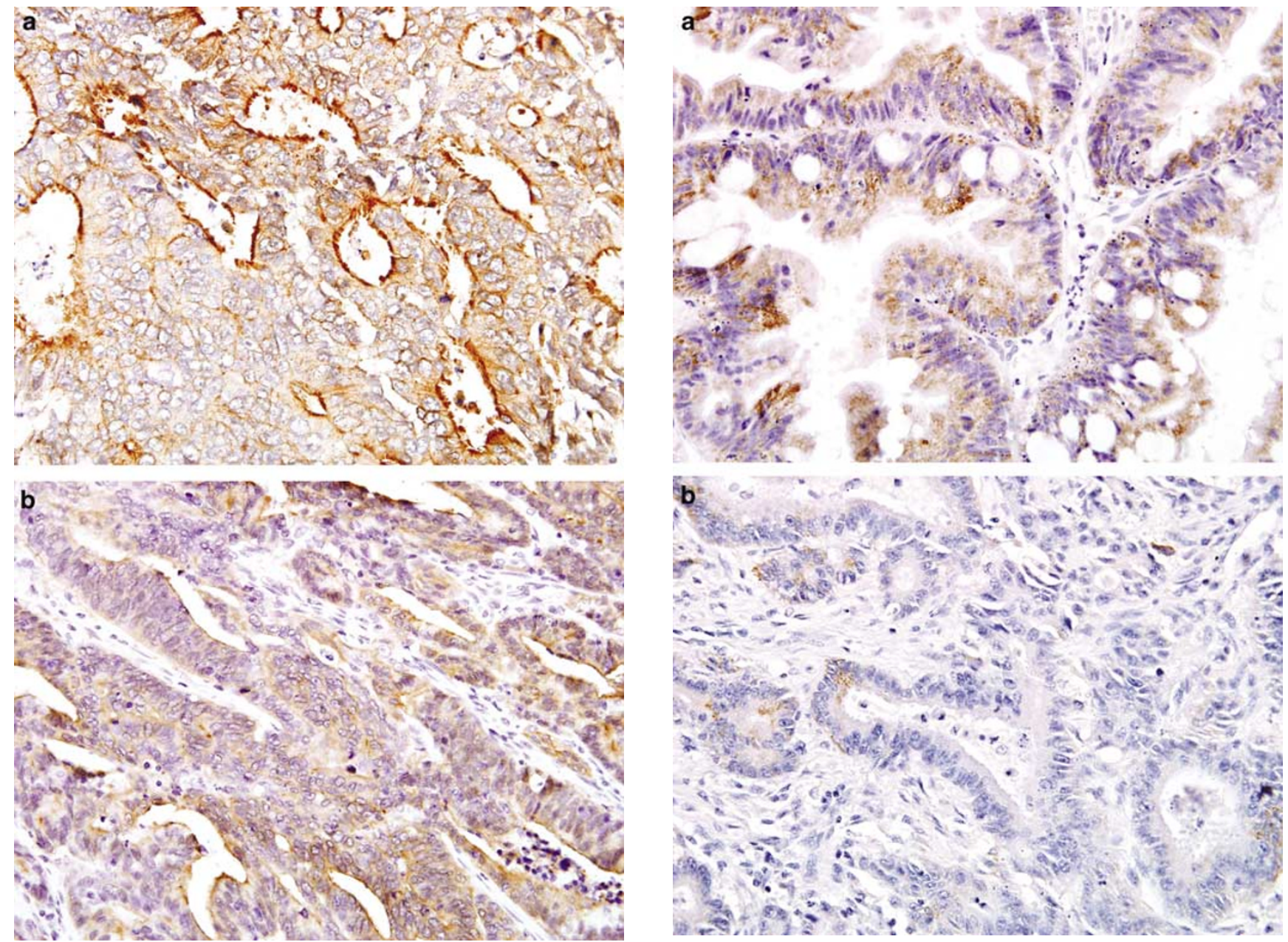

Figure 2 Diffuse cytoplasmic staining with linear apical accentuation for villin seen in a secondary colorectal adenocarcinoma (a). Similar staining pattern was also observed in some of the primary bladder adenocarcinomas (b) (original magnification $\times 400)$.

be extremely challenging, particularly when the diagnostic material is a small biopsy and when the clinical information is incomplete. Currently, there are only a few immunomarkers available for the distinction but their use is limited because a significant proportion of bladder adenocarcinomas share the same immunophenotype with colorectal adenocarcinomas. It is thus desirable to evaluate additional markers with established tissue specificity, to exploit their potential in aiding in the differential diagnosis.

CDX2 is a mammalian homeobox gene encoding a nuclear transcription factor whose expression appears to be strictly limited to the intestinal epithelium. ${ }^{4,5}$ In addition to serve a key role in regulating normal intestinal development and homeostasis, CDX2 may also function as a tumor suppressor., ${ }^{4,6-8}$ In mouse model, reduced expression of CDX2 results in colonic polyposis via the activation of the mTOR pathway, the mammalian target of
Figure 3 Diffuse cytoplasmic staining for AMACR noted in a primary bladder adenocarcinoma (a). Focal positivity was also noted in both primary bladder adenocarcinomas and colorectal adenocarcinomas. The example shown here was a colorectal adenocarcinoma (b) (original magnification $\times 400$ ).

Table 2 Coexpression of CDX2, villin and AMACR in primary bladder adenocarcinoma (PBA), secondary colorectal adenocarcinoma (SCA), primary colorectal adenocarcinoma (PCA), and urothelial carcinoma (UC)

\begin{tabular}{lcccc}
\hline Marker & \multicolumn{4}{c}{ No. (\%) positive } \\
\cline { 2 - 5 } & $\begin{array}{c}P B A \\
(n=17)\end{array}$ & $\begin{array}{c}S C A \\
(n=17)\end{array}$ & $\begin{array}{c}P C A \\
(n=23)\end{array}$ & $\begin{array}{c}U C \\
(n=14)\end{array}$ \\
\hline CDX2/villin & $7(41)$ & $16(94)$ & $23(100)$ & 0 \\
CDX2/AMACR & $5(29)$ & $12(71)$ & $15(65)$ & 0 \\
Villin/AMACR & $8(47)$ & $13(77)$ & $15(65)$ & 0 \\
CDX2/villin/ & $4(24)$ & $12(71)$ & $15(65)$ & 0 \\
AMACR & & & &
\end{tabular}

rapamycin, ${ }^{9}$ that leads to chromosomal instability, accelerated $\mathrm{G}_{1}-\mathrm{S}$ transition, suppression of apoptosis, decreased expression of p27 and increased cyclin E-associated kinase activity. ${ }^{10}$ CDX2 also 
regulates the expression of $\mathrm{p} 21,{ }^{11}$ a cyclin-dependent kinase inhibitor, and $\mathrm{COX}-2,{ }^{12}$ an essential enzyme in prostaglandin synthesis that is often upregulated in colorectal adenocarcinomas.

Despite the earlier findings that the expression of CDX2 was downregulated during colorectal carcinogenesis, ${ }^{13,14}$ recent studies have clearly shown CDX2 to be a highly sensitive marker for colorectal adenocarcinoma. ${ }^{15-18}$ The reported frequency of positive CDX2 immunostaining ranged from 98 to $100 \%$ in three series,,$^{15,17,18}$ and was $86 \%$ in one study where tissue microarrays containing only one core per tumor were used. ${ }^{16}$ The vast majority of the cases exhibited a strong and diffuse staining pattern. Although not entirely specific, the documented high sensitivity makes CDX2 a relatively reliable marker for adenocarcinoma of colorectal origin, which may be helpful in distinguishing metastatic colorectal adenocarcinoma from primary adenocarcinomas of the lung, ${ }^{15,17,19}$ ovary, ${ }^{17,20}$ and uterine cervix. ${ }^{21}$

CDX2 expression in primary bladder adenocarcinoma, a morphological mimic of colorectal adenocarcinoma, has not well been investigated to date. In fact, only three cases of bladder adenocarcinoma (including one urachal carcinoma) have been examined by Werling et $a l,{ }^{18}$ which all showed positive CDX2 nuclear staining. In the current study, we examined 17 cases and demonstrate that although nearly $50 \%$ of primary bladder adenocarcinomas express CDX2 in a similar fashion to colorectal adenocarcinomas, a significant proportion of the cases exhibit negative CDX2 immunoreactivity under the identical immunostaining conditions.

Villin is an actin-binding protein that serves a critical role in the maintenance of the brush border organization..$^{22}$ It is expressed with a relatively high specificity in epithelial cells of the gastrointestinal tract that possess brush border microvilli and in adenocarcinomas derived from these cells. ${ }^{23-25}$ Using a multistep protocol including genomic, proteomic and tissue array profiling, villin was recently identified as the best diagnostic marker that distinguishes colorectal adenocarcinoma from ovarian adenocarcinoma. ${ }^{26}$

The potential use of villin in distinguishing primary bladder adenocarcinoma from colorectal adenocarcinoma has been investigated in a limited number of cases. In the study by Tamboli et $a{ }^{27}$ positive villin immunostaining was observed in all four primary bladder adenocarcinomas and all 13 colorectal adenocarcinomas they examined. The three bladder adenocarcinomas examined by Werling et $a l^{18}$ were also positive for villin expression. By examining a larger number of cases in this study, we show that while the majority of bladder adenocarcinomas stain positive for villin, one-third of the cases exhibit negative immunoreactivity in contrast to only one of 40 colorectal adenocarcinomas included in the study.

AMACR, also known as P504S, is a well-characterized enzyme that serves an essential role in $\beta$ - oxidation of dietary branched-chain fatty acids and bile acid intermediates. ${ }^{28}$ It is a novel immunomarker for prostatic adenocarcinoma and its precursor lesion high-grade prostatic intraepithelial neoplasia. ${ }^{29}$ Recently, AMACR has been shown to be expressed in $69-83 \%$ of colorectal adenocarcinoma, ${ }^{30,31}$ although the underlying mechanism(s) remains to be established. Its expression has also been detected in approximately $30 \%$ of urothelial carcinomas, ${ }^{31,32}$ but has not yet previously been examined in bladder adenocarcinomas. The frequency of positive AMACR immunoreactivity in colorectal adenocarcinomas in our study $(70 \%)$ is comparable to those reported previously. ${ }^{30,31}$ However, a similar frequency $(65 \%)$ is also observed in adenocarcinomas of the bladder origin.

In summary, the data presented in this report extend our previous observations ${ }^{2}$ that primary bladder adenocarcinoma is not entirely identical immunophenotypically to colorectal adenocarcinoma. Since essentially all colorectal adenocarcinomas express CDX2 and nearly all express villin, negative staining of one or both of these two markers strongly suggests a bladder primary in the appropriate clinical setting. This may be particularly true of CDX2 since it appears to be less frequently expressed than villin in bladder adenocarcinomas. Immunostains for CDX2 and villin are thus useful additions to the limited list of diagnostic markers (including CK7, CK20, thrombomodulin and $\beta$ catenin) for the distinction between primary adenocarcinoma of the bladder and secondary colorectal adenocarcinoma. On the contrary, AMACR does not appear to contribute to the differential diagnosis. In the future, it would be of interest to perform gene expression profiling to identify additional markers that are positive in bladder adenocarcinomas but negative in colorectal adenocarcinomas to complement CDX2 and villin.

\section{Acknowledgement}

We thank Ms Prosperidad Amargo for her excellent technical assistance.

\section{References}

1 Bates AW, Baithun SI. Secondary neoplasms of the bladder are histological mimics of nontransitional cell primary tumours: clinicopathological and histological features of 282 cases. Histopathology 2000;36:32-40.

2 Wang HL, Lu DW, Yerian LM, et al. Immunohistochemical distinction between primary adenocarcinoma of the bladder and secondary colorectal adenocarcinoma. Am J Surg Pathol 2001;25:13801387.

3 Dahm P, Gschwend JE. Malignant non-urothelial neoplasms of the urinary bladder: a review. Eur Urol 2003;44:672-681. 
4 Guo RJ, Suh ER, Lynch JP. The role of cdx proteins in intestinal development and cancer. Cancer Biol Ther 2004;3:593-601.

5 Li MK, Folpe AL. CDX-2, a new marker for adenocarcinoma of gastrointestinal origin. Adv Anat Pathol 2004;11:101-105.

6 Bonhomme C, Duluc I, Martin E, et al. The Cdx2 homeobox gene has a tumour suppressor function in the distal colon in addition to a homeotic role during gut development. Gut 2003;52:1465-1471.

7 Mutoh H, Sakurai S, Satoh K, et al. Development of gastric carcinoma from intestinal metaplasia in Cdx2transgenic mice. Cancer Res 2004;64:7740-7747.

8 Sivagnanasundaram S, Islam I, Talbot I, et al. The homeobox gene CDX2 in colorectal carcinoma: a genetic analysis. Br J Cancer 2001;84:218-225.

9 Asnaghi L, Bruno P, Priulla M, et al. mTOR: a protein kinase switching between life and death. Pharmacol Res 2004;50:545-549.

10 Aoki K, Tamai Y, Horiike S, et al. Colonic polyposis caused by mTOR-mediated chromosomal instability in $A p c^{+/ \Delta 716} \mathrm{Cdx} 2^{+/-}$compound mutant mice. Nat Genet 2003;35:323-330.

11 Bai YQ, Miyake S, Iwai T, et al. CDX2, a homeobox transcription factor, upregulates transcription of the p21/WAF1/CIP1 gene. Oncogene 2003;22: 7942-7949.

12 Kim SP, Park JW, Lee SH, et al. Homeodomain protein CDX2 regulates COX-2 expression in colorectal cancer. Biochem Biophys Res Commun 2004;315:93-99.

13 Ee HC, Erler T, Bhathal PS, et al. Cdx-2 homeodomain protein expression in human and rat colorectal adenoma and carcinoma. Am J Pathol 1995;147: 586-592.

14 Mallo GV, Rechreche H, Frigerio JM, et al. Molecular cloning, sequencing and expression of the mRNA encoding human Cdx1 and Cdx2 homeobox: downregulation of $\mathrm{Cdx} 1$ and $\mathrm{Cdx} 2$ mRNA expression during colorectal carcinogenesis. Int J Cancer 1997;74:35-44.

15 Barbareschi M, Murer B, Colby TV, et al. CDX-2 homeobox gene expression is a reliable marker of colorectal adenocarcinoma metastases to the lungs. Am J Surg Pathol 2003;27:141-149.

16 Kaimaktchiev V, Terracciano L, Tornillo L, et al. The homeobox intestinal differentiation factor CDX2 is selectively expressed in gastrointestinal adenocarcinomas. Mod Pathol 2004;17:1392-1399.

17 Moskaluk CA, Zhang H, Powell SM, et al. Cdx2 protein expression in normal and malignant human tissues: an immunohistochemical survey using tissue microarrays. Mod Pathol 2003;16:913-919.

18 Werling RW, Yaziji H, Bacchi CE, et al. CDX2, a highly sensitive and specific marker of adenocarcinomas of intestinal origin: an immunohistochemical survey of 476 primary and metastatic carcinomas. Am J Surg Pathol 2003;27:303-310.

19 Saad RS, Cho P, Silverman JF, et al. Usefulness of $\mathrm{Cdx} 2$ in separating mucinous bronchioloalveolar adenocarcinoma of the lung from metastatic mucinous colorectal adenocarcinoma. Am J Clin Pathol 2004;122: 421-427.

20 Logani S, Oliva E, Arnell PM, et al. Use of novel immunohistochemical markers expressed in colonic adenocarcinoma to distinguish primary ovarian tumors from metastatic colorectal carcinoma. Mod Pathol 2004, Epub ahead of print.

21 Raspollini MR, Baroni G, Taddei A, et al. Primary cervical adenocarcinoma with intestinal differentiation and colonic carcinoma metastatic to cervix: an investigation using $\mathrm{Cdx}-2$ and a limited immunohistochemical panel. Arch Pathol Lab Med 2003;127: 1586-1590.

22 Athman R, Louvard D, Robine S. The epithelial cell cytoskeleton and intracellular trafficking. III. How is villin involved in the actin cytoskeleton dynamics in intestinal cells? Am J Physiol Gastrointest Liver Physiol 2002;283:G496-G502.

23 Bacchi CE, Gown AM. Distribution and pattern of expression of villin, a gastrointestinal-associated cytoskeletal protein, in human carcinomas: a study employing paraffin-embedded tissue. Lab Invest 1991; 64:418-424.

24 Moll R, Robine S, Dudouet B, et al. Villin: a cytoskeletal protein and a differentiation marker expressed in some human adenocarcinomas. Virchows Arch B Cell Pathol Incl Mol Pathol 1987;54:155-169.

25 West AB, Isaac CA, Carboni JM, et al. Localization of villin, a cytoskeletal protein specific to microvilli, in human ileum and colon and in colonic neoplasms. Gastroenterology 1988;94:343-352.

26 Nishizuka S, Chen ST, Gwadry FG, et al. Diagnostic markers that distinguish colon and ovarian adenocarcinomas: identification by genomic, proteomic, and tissue array profiling. Cancer Res 2003;63:5243-5250.

27 Tamboli P, Mohsin SK, Hailemariam S, et al. Colonic adenocarcinoma metastatic to the urinary tract versus primary tumors of the urinary tract with glandular differentiation: a report of 7 cases and investigation using a limited immunohistochemical panel. Arch Pathol Lab Med 2002;126:1057-1063.

28 Ferdinandusse S, Denis S, IJlst L, et al. Subcellular localization and physiological role of $\alpha$-methylacylCoA racemase. J Lipid Res 2000;41:1890-1896.

29 Jiang Z, Woda BA, Wu CL, et al. Discovery and clinical application of a novel prostate cancer marker: $\alpha$ methylacyl CoA racemase (P504S). Am J Clin Pathol 2004;122:275-289.

30 Jiang Z, Fanger GR, Banner BF, et al. A dietary enzyme: $\alpha$-methylacyl-CoA racemase/P504S is overexpressed in colon carcinoma. Cancer Detect Prev 2003;27:422-426.

31 Zhou M, Chinnaiyan AM, Kleer CG, et al. Alphamethylacyl-CoA racemase: a novel tumor marker over-expressed in several human cancers and their precursor lesions. Am J Surg Pathol 2002;26:926-931.

32 Jiang Z, Fanger GR, Woda BA, et al. Expression of $\alpha$ methylacyl-CoA racemase (P504s) in various malignant neoplasms and normal tissues: a study of 761 cases. Hum Pathol 2003;34:792-796. 